Graziele G. Bovi, Oluwafemi J. Caleb, Eylin Klaus, Filip Tintchev, Cornelia Rauh, Pramod V. Mahajan

\title{
Moisture absorption kinetics of FruitPad for packaging of fresh strawberry
}

Journal article | Accepted manuscript (Postprint)

This version is available at https://doi.org/10.14279/depositonce-9720

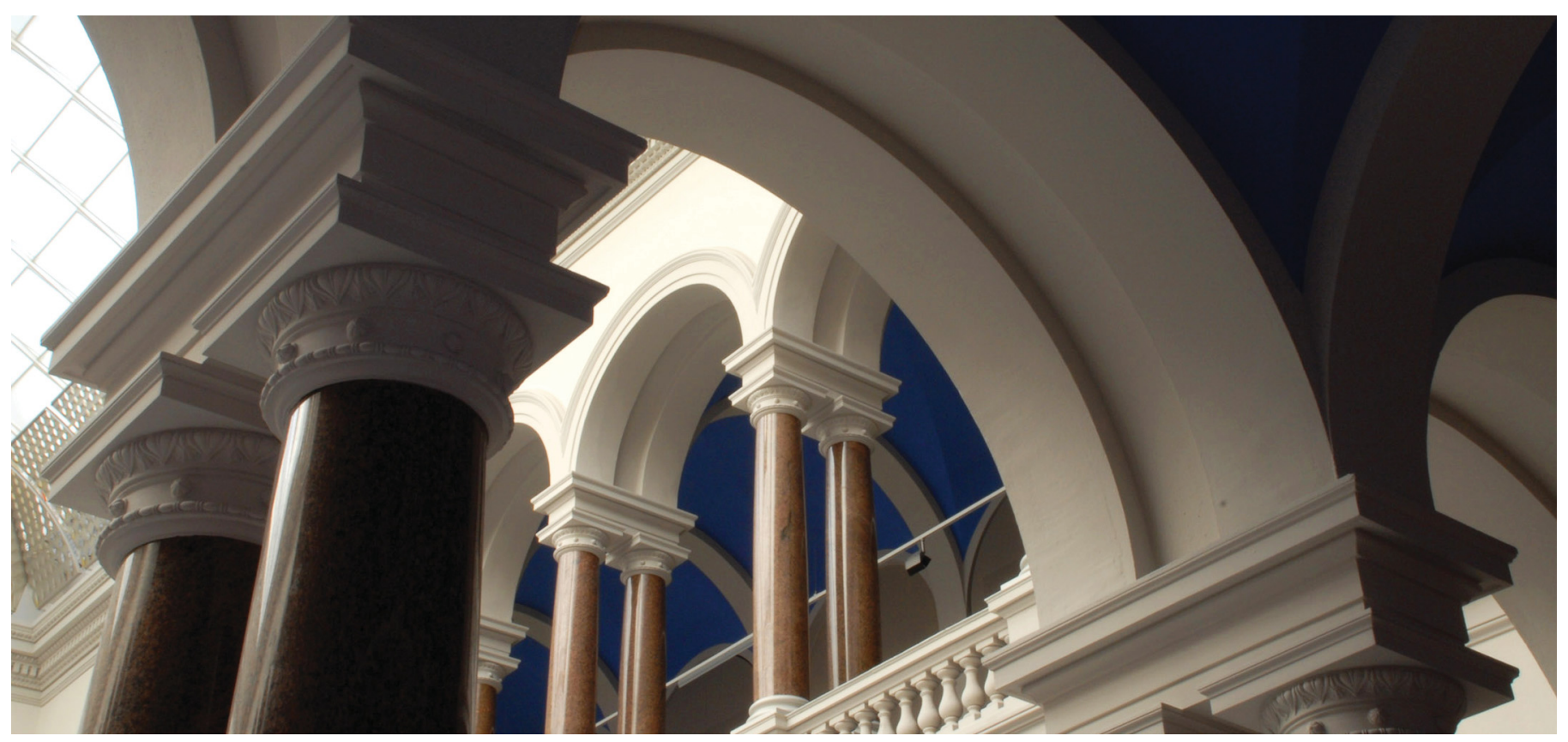

Bovi, G. G., Caleb, O. J., Klaus, E., Tintchev, F., Rauh, C., \& Mahajan, P. V. (2018). Moisture absorption kinetics of FruitPad for packaging of fresh strawberry. Journal of Food Engineering, 223, 248-254. https://doi.org/10.1016/j.jfoodeng.2017.10.012 
1 Moisture absorption kinetics of FruitPad for packaging of fresh strawberry

2

3 In: Journal of Food Engineering, 223, 248-254.

4

5

6 Cite as: Bovi, G. G., Caleb, O. J., Klaus, E., Tintchev, F., Rauh, C., \& Mahajan, P. V. (2018).

7 Moisture absorption kinetics of FruitPad for packaging of fresh strawberry. Journal of Food

8 Engineering, 223, 248-254.

9 doi: https://doi.org/10.1016/j.jfoodeng.2017.10.012

10

11

12

13

14

15

16

17

18

19

20

21

22

23

24

25 


\section{Moisture Absorption Kinetics of FruitPad for Packaging of Fresh Strawberry}

Graziele G. Bovi ${ }^{\text {a, c }}$, Oluwafemi J. Caleb ${ }^{\text {a, b }}$, Eylin Klaus ${ }^{\text {d }}$, Filip Tintchev ${ }^{\text {d }}$, Cornelia Rauh ${ }^{\text {c }}$, Pramod V. Mahajan ${ }^{a^{*}}$

${ }^{a}$ Department of Horticultural Engineering, Leibniz Institute for Agricultural Engineering and Bioeconomy (ATB), Potsdam, Germany

${ }^{\mathrm{b}}$ Post-harvest and Agro-processing Technologies, Agricultural Research Council (ARC), InfruitecNietvoorbij, Stellenbosch, South Africa

${ }^{c}$ Department of Food Biotechnology and Food Process Engineering, Technical University of Berlin, Germany

${ }^{\mathrm{d}}$ McAirlaid's Vliesstoffe GmbH, Berlingerode, Germany

*Corresponding author: Phone: +49 3315699615

E-mail: pmahajan@atb-potsdam.de(Pramod V. Mahajan)

\section{Abstract}

This study analysed the moisture absorption kinetics of FruitPad embedded with different concentrations of fructose with further application of such pads in packaging of fresh strawberries. The FruitPad was exposed to different storage conditions (temperature and $\mathrm{RH}$ ) and moisture absorption kinetics was gravimetrically determined over 5 days of storage. FruitPad with $30 \%$ fructose showed highest amount of moisture absorption $\left(0.94 \mathrm{~g}\right.$ of water/g of pad) at $20{ }^{\circ} \mathrm{C}$ and $100 \%$ RH. The Weibull model combined with the Flory-Huggins model adequately described changes in moisture content of the FruitPad with respect to storage time and humidity $\left(\mathrm{R}^{2}=93-\right.$ 96\%). The FruitPad containing fructose minimized in-package condensation compared to the pad without fructose. Weight loss of packaged strawberry was less than $0.9 \%$ which was much below the acceptable limit of $6 \%$ for strawberry.

Keywords: Modified atmosphere packaging, Fragaria $x$ ananassa Duch, condensation, absorbing pads 


\section{Introduction}

Fresh fruits and vegetables $(\mathrm{FF} \& \mathrm{~V})$ have continuous metabolism as they keep losing water due to respiration and transpiration processes. If not controlled, water released through these processes results in moisture condensation inside packaged $F F \& V$; since packaging acts as an additional barrier for moisture transfer (Bovi et al., 2016). In turn, condensation represents a risk to product quality as water may accumulate in packaging system and/or on product surface leading to defects in external appearance, quality deterioration, flavour loss, and promoting growth of spoilage microorganisms (Linke and Geyer, 2013). Thus, moisture regulation is essential for extending FF\&V shelf life as it can lessen the risk of spoilage causing microorganisms growth, and therefore maintain product quality. Various strategies for controlling moisture inside packaged fresh produce have been reported: i) use of moisture absorbers inside the package (Mahajan et al., 2008); ii) use of a humidity-regulating tray that can actively absorb moisture (Rux et al., 2016) ; and, iii) use of a packaging material with a very high permeability for water vapour (Caleb et al., 2016).

Moisture absorbing pads are one of the most innovative and versatile applications of active food packaging systems. It is generally constituted of an upper and lower sheet of film coating and a core middle layer composed mainly of cellulose and an active ingredient that absorbs excess liquid (drip loss) present in the package. Pads can be divided into two main categories: water contact and noncontact absorber. The water contact absorber pad is commercially being used for packaging of meat products, such as fish, beef, and pork (Fang et al., 2017). These pads are useful, however; the excess moisture leached out from the product must be in direct contact with the active ingredient of the pad in order to be absorbed. Therefore, these pads are not suitable for fresh produce application as $\mathrm{FF} \& \mathrm{~V}$ continue to respire and transpire and the water vapour released in these process remains inside the package headspace and not necessarily in direct contact with the pad. Thus, there is a need for novel and non-contact moisture absorbing pads that can not only absorb the water in direct contact with FF\&V but also water vapour from the package headspace.

The idea of incorporating active hygroscopic $\mathrm{NaCl}$ between the two layers, like humidity regulating tray (Rux et al., 2016), was further applied to absorbing pads using fructose as an active ingredient. Fructose contributes to functional attributes when applied to food and beverage. These include flavour enhancement, osmotic stability, humectancy, and freezing point depression (White, 2014). These functional properties may be attributed to physical and chemical properties of fructose itself or to the interaction of fructose with the food system. Fructose is hygroscopic and can absorb moisture from its environment. It begins to absorb water vapour at approximately $55 \%$ relative humidity $(\mathrm{RH})$. Furthermore, fructose has good humectant properties and it can retain moisture for a long period of time, even at low RH (White, 2014). Therefore, fructose has a great potential of 
acting as a moisture absorber. The integration of fructose into the matrix of absorbing pad structures, as active substance, is promising as it can absorb free water in the tray and also absorb excess water vapour in the package headspace. In this context, the aim of this study was to investigate the moisture absorption kinetics of absorbing pads (namely FruitPad) matrix, embedded with varying concentrations of fructose as active ingredient for moisture absorption.

\section{Materials and methods}

\subsection{FuitPad}

The pad consisted of a 3-layer structure (Fig. 1). The top and bottom layers were made of polyethylene with 8 micro-perforations of $0.3 \mathrm{~mm}$ diameter per $\mathrm{cm}^{2}$. The middle layer contained cellulose fibres (McAirLaid's Vliesstoffe GmbH, Steinfurt, Germany). These FruitPads (FruitPad00) were incorporated with two concentrations of fructose (20 and $30 \%$, henceforth called FruitPad20 and FruitPad30, respectively in the manuscript) in the middle layer using the commercial production facilities of McAirlaid's Vliesstoffe $\mathrm{GmbH}$. The remaining matrix consisted of $28 \%$ film and $52 \%$ cellulose (for $20 \%$ fructose pad), and $21 \%$ film and $49 \%$ cellulose (for $30 \%$ fructose pad).

\subsection{Moisture absorption kinetics}

Pad samples $(10.3 \times 7.5 \mathrm{~cm})$, in triplicate, were stored in $190 \mathrm{~L}$ metal chambers at temperatures 4, 12 , and $20{ }^{\circ} \mathrm{C}$. The $\mathrm{RH}$ was maintained at 76, 86, 96 and $100 \% \mathrm{RH}$ by using saturated salts solutions (Rux et al., 2016). The water vapour absorption of the FruitPad was gravimetrically determined by measuring increase in weight of the pads at regular intervals for 5 days using an electronic balance (Sartorius, Göttingen, Germany). The moisture content of the FruitPad was expressed as shown in Eq. (1).

$$
M_{t}=\left(\frac{W_{t}-W_{i}}{W_{i}}\right)
$$

where $M_{t}$ is the moisture content of the FruitPad at time $t$ ( $g$ water $g^{-1}$ pad), $t$ is time $(h), W_{i}$ and $W_{t}$ are the weight of the FruitPad $(\mathrm{g})$ in the beginning and at time $\mathrm{t}$, respectively.

Weibull model has been shown to be a suitable model to describe moisture absorption as a function of time (Mahajan et al., 2008; Rux et al., 2016), and therefore was used in this study, as a primary model, to describe the curves of moisture content versus time as shown in Eq. (2):

$$
M_{t}=M_{0}+\left(M_{\infty}-M_{0}\right) x\left[1-e^{\left(\frac{-t}{\beta 1}\right)}\right]
$$


where $\mathrm{M}_{\mathrm{o}}$ is the initial moisture content of the FruitPad ( $\mathrm{g}$ water $\mathrm{g}^{-1}$ pad), which is zero as the FruitPad was dry, $\mathrm{M}_{\infty}$ is the moisture holding capacity ( $\mathrm{g}$ water $\mathrm{g}^{-1}$ pad) at equilibrium, and $\beta_{1}$ is the kinetic parameter that defines the rate of moisture uptake process and represents the time needed to accomplish approximately $63 \%$ of the moisture uptake process. Furthermore, $\mathrm{M}_{\infty}$ can take infinite time to be measured; however, the Weibull model offers the possibility of estimating the $\mathbf{M}_{\infty}$ with experimental data of moisture content with time.

\subsection{Packaging of strawberry}

Strawberries (cv. Flair) were obtained from a commercial grower (Karls Erlebnis-Dorf Elstal, Germany). They were precooled to the study temperature for 3 hours. Polypropylene tray (16 x 12 x $5 \mathrm{~cm}$ ) was used to pack 15 strawberries of $260 \pm 5 \mathrm{~g}$. It was covered with bi-axially oriented polypropylene Propafilm ${ }^{\mathrm{TM}} \mathrm{RGP} 25$ (25 mm thickness; permeability rate to $\mathrm{O}_{2}, 8.5 \times 10^{-12} \mathrm{~mol} \mathrm{~m}^{-2} \mathrm{~s}^{-1}$ $\mathrm{Pa}^{-1}$ at $23{ }^{\circ} \mathrm{C}$ and $0 \% \mathrm{RH}$; water vapour, $5.7 \times 10^{-6} \mathrm{~mol} \mathrm{~m}^{-2} \mathrm{~s}^{-1} \mathrm{~Pa}^{-1}$ at $23{ }^{\circ} \mathrm{C}$ and $85 \% \mathrm{RH}$ ). The lid film was perforated with 2 micro-perforations of diameter $0.7 \mathrm{~mm}$. Packages were stored for 5 days at $12{ }^{\circ} \mathrm{C}$. Packages were named FruitPad00 for the pad containing 0\% of fructose, FruitPad20 for the pad with $20 \%$ of fructose, FruitPad30 for the package with $30 \%$ of fructose, and control for the package without FruitPad. Two replicates of each package were performed.

\subsection{Package performance evaluation}

Weight loss was determined by weighing the strawberries at the beginning of the experiment and after storage. The FruitPad absorption capacity was calculated by weight of the FruitPad on day 0 and day 5. The amount of water vapour condensed inside the package was quantified by weighing the package and film before and after the condensed water was removed.

\subsection{Statistical analysis}

The constants of all the presented models were obtained by fitting the experimental data into the equations by using regression analysis and Solver tool in Microsoft Excel (Office 2010, Microsoft, Germany). The statistical analysis was carried out using Statistica software (version 10.0, StatSoft Inc., Tulsa, USA).

\section{Results and discussion}

\subsection{Moisture absorption kinetics}

Moisture uptake increased significantly ( $\mathrm{p}<0.05$ ) over storage time (Fig. 2). Generally, moisture uptake for all FruitPads was faster on the first day and substantially slower from day 2. FruitPad kept at higher humidities had higher moisture absorption capacity in comparison to lower 
humidities at the end of day 5. At $20{ }^{\circ} \mathrm{C}$, FruitPad30 absorbed $0.94 \mathrm{~g}$ water $\mathrm{g}^{-1}$ pad at $100 \% \mathrm{RH}$ and $0.13 \mathrm{~g}$ water $\mathrm{g}^{-1}$ pad at $76 \% \mathrm{RH}$, an increase of 7.2 times on water uptake. Results are consistent with other studies reported as it is well established that there is higher moisture uptake at higher humidity for a diverse range of materials. For instance, Saberi et al. (2016) reported that the slope of the isotherms for a pea starch films was smaller at lower $a_{w}$ (less than 0.60), and with a rising in $\mathrm{a}_{\mathrm{w}}$ the slope increased quickly.

Fig. 3 shows the effect of fructose concentration and storage $\mathrm{RH}$ on the total moisture content $\left(\mathrm{M}_{\mathrm{t}}\right)$.

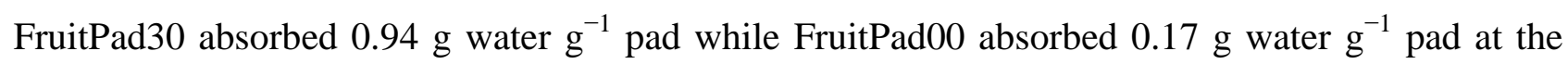
same humidity and temperature $\left(100 \% \mathrm{RH}\right.$ and $\left.20^{\circ} \mathrm{C}\right)$. It is clear that the concentration of fructose, as well as the $\mathrm{RH}$, had a significant impact on $\mathrm{M}_{\mathrm{t}}$. In addition, results showed that incorporation of fructose into the FruitPad increased the water vapour absorption of the pads. One of the reasons for this could be due to the high hygroscopic property of fructose. Fructose is highly soluble in water $\left(3.75 \mathrm{~g} / \mathrm{mL}\right.$ at $20{ }^{\circ} \mathrm{C}$ ) (Chemical Book, 2017). Hence, it keeps absorbing moisture even after the powder form of fructose turns into liquid form. The resultant fructose-water solution is very viscous (Silva et al., 2009), and can be easily retained by the cellulose fibres of the FruitPad. Therefore, the higher amount of fructose per gram of FruitPad, the higher is the potential for moisture absorption. Similar result was found in a study with humidity-regulating trays incorporated with salt as the active compound (Rux et al., 2016).

\subsection{Model development}

With the results obtained from the moisture absorption kinetics a primary model based on the Weibull model was developed for each FruitPad at each RH and temperature. Table 1 showed the primary model parameters obtained at $12{ }^{\circ} \mathrm{C}$. As can be seen $\mathrm{M}_{\infty}$ was clearly affect by the increase in $\mathrm{RH}$ and fructose concentration. In addition, results showed that $\mathrm{RH}$ and fructose concentration had a significant impact $(\mathrm{p}<0.05)$ on moisture absorption; however temperature did not (Fig. 4a).

As RH had an impact, the Flory-Huggins model (Eq.3) was then employed to relate the moisture holding capacity (g water $\mathrm{g}^{-1}$ pad) at equilibrium $(\mathrm{M} \infty)$ with RH (Saberi et al., 2016).

$$
M_{\infty}=A x \quad e^{\left(B \times a_{w}\right)}
$$

where $\mathrm{a}_{\mathrm{w}}$ is the water activity $(\mathrm{RH} / 100)$; and A and $\mathrm{B}$ are model constants.

Eq. (3) was then combined with Eq. (2) yielding in a secondary model (Eq. 4), in order to express the influence of $\mathrm{RH}$ in $\mathrm{M}_{\infty}$.

$$
M_{t}=M_{0}+\left(A x e^{\left(B x a_{w}\right)}-M_{0}\right) x\left[1-e^{\left(\frac{-t}{\beta 2}\right)}\right]
$$


Therefore, a secondary model for each fructose concentration was developed taking into account $\mathrm{RH}$ and fructose concentration and not the temperature effect. This model was then used to fit the experimental data at all $\mathrm{RH}$ and temperature for each fructose concentration. The secondary model parameters and the coefficient of determination $\left(\mathrm{R}^{2}\right)$ for each combination are shown in Table 2. Results showed that the Weibull model combined with the Flory-Huggins model adequately described changes in moisture content of the FruitPad with respect to storage time $\left(R^{2}=93-96 \%\right)$. Predicting the moisture content of the FruitPad is of considerable importance when designing optimal packaging systems. Every fresh produce gives out different amounts of water due to the respiration and transpiration process; therefore, for every product there is a different requirement for selecting the most suitable moisture absorber (Bovi and Mahajan, 2017). For this reason it is important to know how much moisture each FruitPad can absorb so that retailers can choose which fructose concentration is more suitable for each given fresh produce. In addition, Fig. $4 \mathrm{~b}$ shows the experimental $v s$ predicted values of the equilibrium moisture content $\left(\mathrm{M}_{\infty}\right)$ of the secondary model for all concentrations of fructose.

\subsection{Package performance evaluation}

Strawberry weight loss was significantly influenced by the FruitPad inside the package (Fig. 5). Tukey's test showed that there was no significant difference in weight loss between the control and the FruitPad00 sample, whereas significant difference in weight loss was observed between the control and pads embedded with fructose $(\mathrm{p}<0.05)$. Overall, percentage weight loss were significantly below the recommended maximum acceptable of 6\% (Nunes and Emond, 2007). This showed that MAP played a significant role in minimizing the weight loss of strawberries. Furthermore, it is noteworthy that weight loss includes both water and carbon loss. Water loss is attributed to transpiration, while carbon loss is due to respiration (Saltveit, 1996). However, in this study the carbon loss was considered as negligible and water loss via transpiration was considered as the main driver of the weight loss.

In addition, the very low weight loss for MA-packaged strawberries samples could be attributed to the higher water vapour barrier property of the BOPP film, which resulted in a higher RH inside the package (Caleb et al., 2016). However, part of the moisture released by the product probably escaped the packaging material through the optimized film micro-perforations (based on preliminary study) for gas exchange. This contributed to very low condensation (less than $0.02 \mathrm{~g}$ ) underneath the packaging film (Fig. 5), which was beneficial for maintaining the quality of the strawberries. Nevertheless, the use of pads did not avoid the formation of water condensation but it might have reduced the volume. The presence of water condensation could be attributed to the transpiration rate of the strawberries, which was higher than the absorption rate of the FruitPad. 
Furthermore, water absorbed by the FruitPad was proportional to the concentration of fructose present in the FruiPad. The highest moisture gain was found in FruitPad30 (1.16 $\mathrm{g}$ of water $\mathrm{g}^{-1}$ of

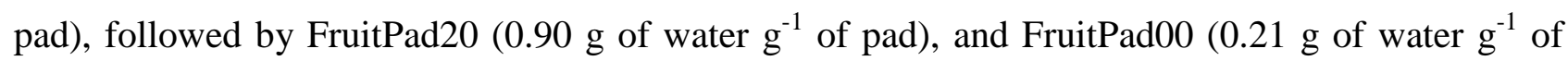
pad). This behavior was also observed in the moisture sorption kinetics of the FruitPad. Fructose has the functional attribute of hygroscopicity and humectancy, which means it has the ability to bind and hold moisture (White, 2014). Therefore, higher concentration of fructose leads to higher moisture uptake. This trend was also seen in the study carried out by Rux et al. (2016). In their study, humidity trays were developed with two concentrations of $\mathrm{NaCl} 0 \mathrm{wt} \%$ (T-0) and $12 \mathrm{wt} \%$ (T12) as active compound of the humidity regulating trays and were tested with strawberries stored at $13{ }^{\circ} \mathrm{C}$ for 7 days. The total amount of strawberry moisture loss ranged from 1.6 to $7.9 \mathrm{~g}$ for strawberries, with the samples packed in the control-PP trays losing the least amount of water (1.6 $\mathrm{g}$; $0.6 \%$ of total strawberry weight), followed by $\mathrm{T}-0(6.0 \mathrm{~g}, 2.2 \%$ of total strawberry weight), and T12 trays losing the most $(7.9 \mathrm{~g}, 2.9 \%$ of total strawberry weight). These results also show that the use of $\mathrm{NaCl}$ as active compound leads to higher weight loss when compared to the use of fructose. In the present study the moisture loss by the strawberry was not higher that $0.92 \%$ of the total strawberry weight. Thus, this shows the possibility to further optimize strategies for in-package moisture absorption. For instance, it is possible to further develop humidity regulating packaging systems by incorporating different proportions and types of active compounds. Overall results showed that FruitPad containing fructose were effective in absorbing water vapour from the package headspace at $12{ }^{\circ} \mathrm{C}$. Furthermore, concentration of fructose integrated into the absorbent pads is product specific and has to be optimised considering the transpiration rate of each fruit or vegetable. If fructose concentration is too high drying of the product surface can occur, and, if it is too low the effects of accumulated condensation will be significant.

\section{Conclusion}

This study showed that both fructose concentration and storage $\mathrm{RH}$ had an effect on the equilibrium moisture content of the FruitPad stored at different temperatures. The Weibull model in combination with the Flory-Huggins model adequately described the changes in moisture content of the pads with respect to storage time $\left(R^{2}>93 \%\right)$. FruitPad containing fructose was effective in absorbing water vapour from the package headspace containing strawberries.

\section{Acknowledgement}

This work was supported by $\mathrm{CNPq}$ through a PhD grant (201623/2015-3). The Alexander von Humboldt Foundation is also appreciated. 


\section{References}

248 Bovi, G.G., Caleb, O.J., Linke, M., Rauh, C., Mahajan, P.V., (2016). Transpiration and moisture evolution in packaged fresh horticultural produce and the role of integrated mathematical models: A review.

250 Biosystems Engineering 150, 24-39.

251 Bovi, G.G., Mahajan, P., (2017). Regulation of humidity in fresh produce packaging. In: Reference Module in 252 Food Science, Elsevier, 1-6.

253 Caleb, O.J., Ilte, K., Fröhling, A., Geyer, M., Mahajan, P.V., (2016). Integrated modified atmosphere and 254 humidity package design for minimally processed Broccoli (Brassica oleracea L. var. italica). Postharvest 255 Biology and Technology 121, 87-100.

256 Chemical Book, (2017). 57-48-7 CAS MSDS (D(-)-Fructose): D(-)-Fructose Water Solubility Property. 257 www.chemicalbook.com/ChemicalProductProperty_EN_CB6139083.htm.

258 Fang, Z., Zhao, Y., Warner, R.D., Johnson, S.K., (2017). Active and intelligent packaging in meat industry. 259 Trends in Food Science \& Technology 61, 60-71.

260 Linke, M., Geyer, M., (2013). Condensation dynamics in plastic film packaging of fruit and vegetables. 261 Journal of Food Engineering 116(1), 144-154.

262 Mahajan, P.V., Rodrigues, F.A.S., Motel, A., Leonhard, A., (2008). Development of a moisture absorber for 263 packaging of fresh mushrooms (Agaricus bisporous). Postharvest Biology and Technology 48(3), 408-414.

264 Nunes, C.N., Emond, J.P., (2007). Relationship between weight loss and visual quality of fruits and 265 vegetables. Proceedings of the Florida State Horticultural Society 120, 235-245.

266 Rux, G., Mahajan, P.V., Linke, M., Pant, A., Sängerlaub, S., Caleb, O.J., Geyer, M., (2016). Humidity267 Regulating Trays: Moisture Absorption Kinetics and Applications for Fresh Produce Packaging. Food and 268 Bioprocess Technology 9(4), 709-716.

269 Saberi, B., Vuong, Q., Chockchaisawasdee, S., Golding, J., Scarlett, C., Stathopoulos, C., (2016). Water 270 Sorption Isotherm of Pea Starch Edible Films and Prediction Models. Foods 5(1), 1-18.

271 Saltveit, M.E., (1996). Physical and physiological changes in minimally processed fruits and vegetables. 272 Tomás-Barberán, F.A. (Ed.), Phytochemistry of Fruit and Vegetables. Oxford University Press, New York. 273 Silva, A.; Brito, A.; Giulietti, M., (2009). Fructose Solubility in Water and Ethanol/Water.Abstracts of the 2742009 AIChE Meeting, Nashville, TN, 2009;AIChE: New York.

275 White, J.S., (2014). Sucrose, HFCS, and Fructose: History, Manufacture, Composition, Applications, and 276 Production, in: Rippe, J.M. (Ed.), Fructose, High Fructose Corn Syrup, Sucrose and Health. Springer New 277 York, New York, NY, 13-33. 
(a)
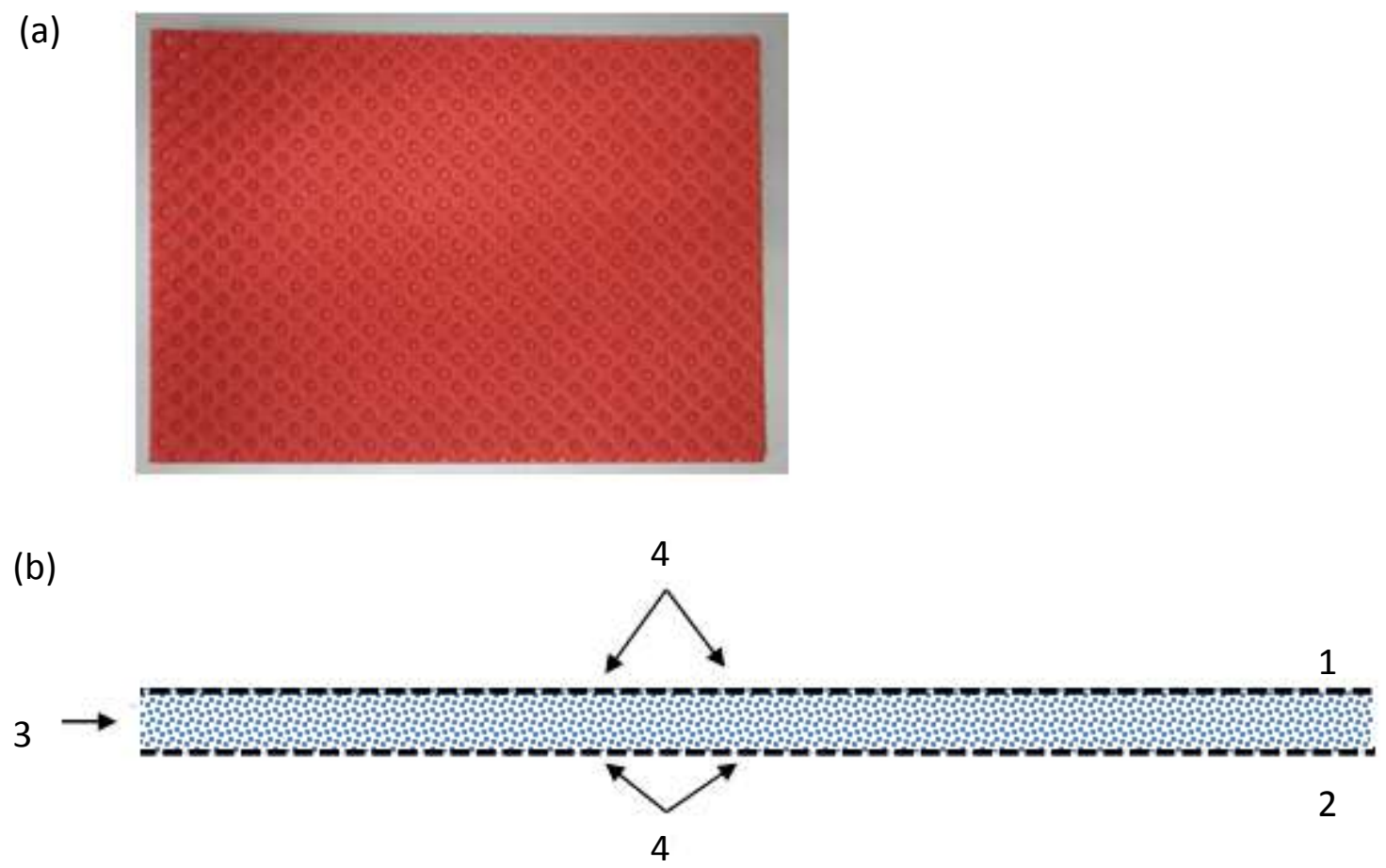

287 Fig 1. Annotated diagram of FruitPad from McAirlaid's Vliesstoffe GmbH. (a) Upper view of the 288 FruitPad (b) Schematic lateral view representation of the FruitPad: 1 - Top layer film, 2 - bottom 289 layer film, 3 - active layer: fructose (blue) and cellulose (white), and 4 - micro-perforations. 


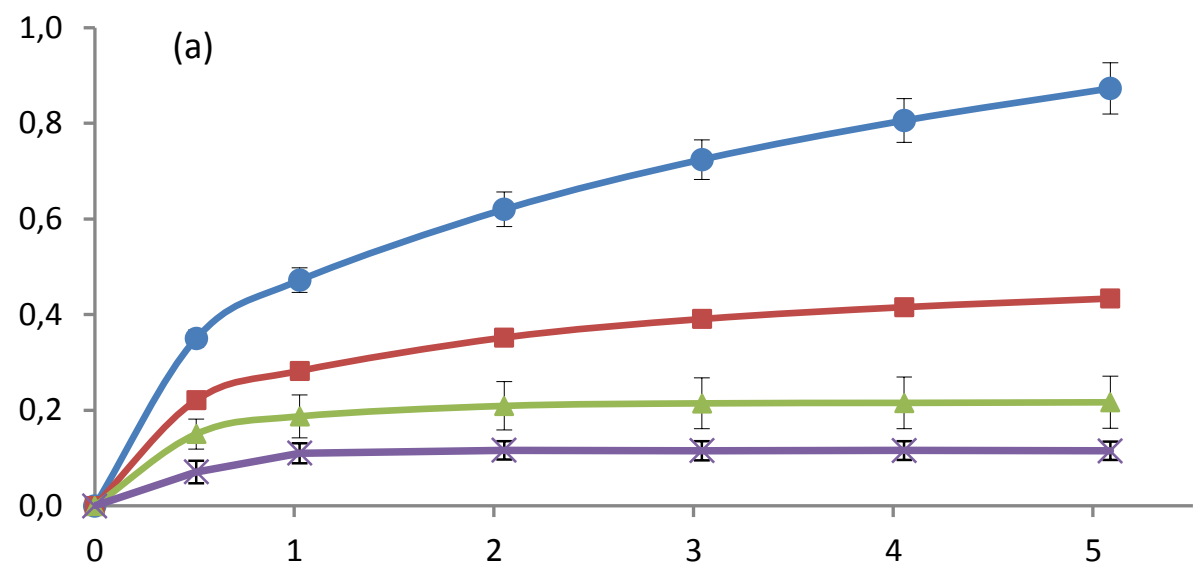

(b)
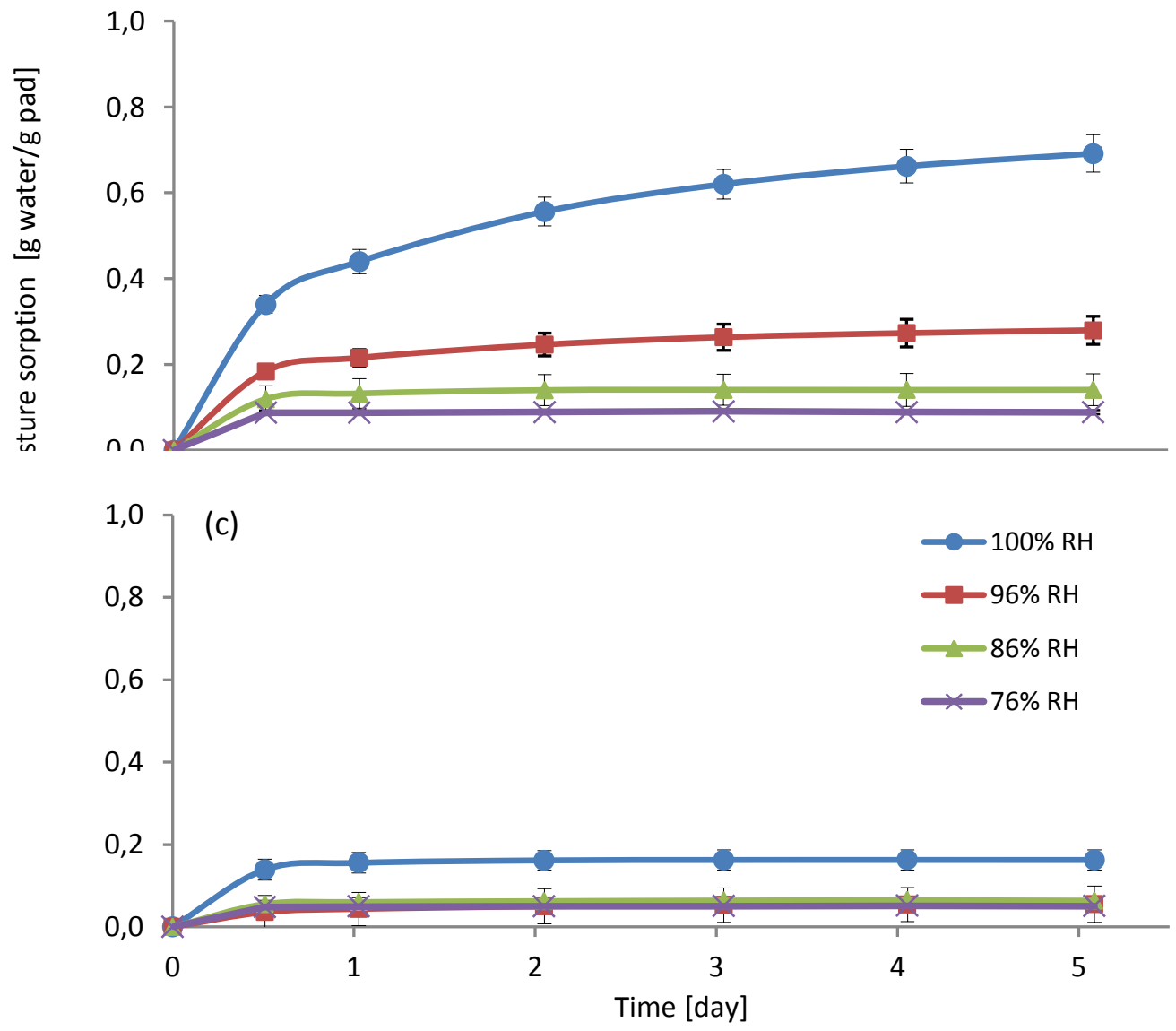

Fig 2. Moisture sorption kinetics of FruitPad stored under different relative humidity at $12{ }^{\circ} \mathrm{C}$ and containing different concentration of fructose (a) FruitPad30 (30\% of fructose), (b) FruitPad20 (20\% of fructose), (c) FruitPad00 ( $0 \%$ of fructose). Error bars represent standard deviation (SD) of mean values $(n=3)$. 


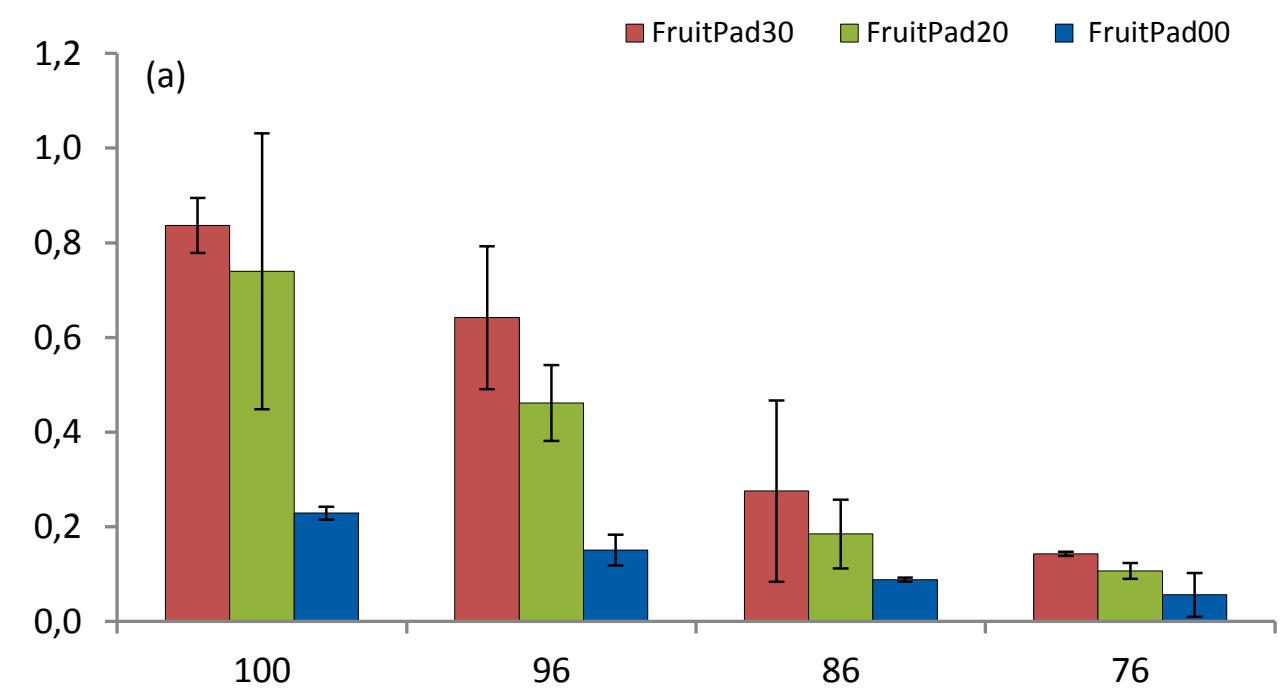

333

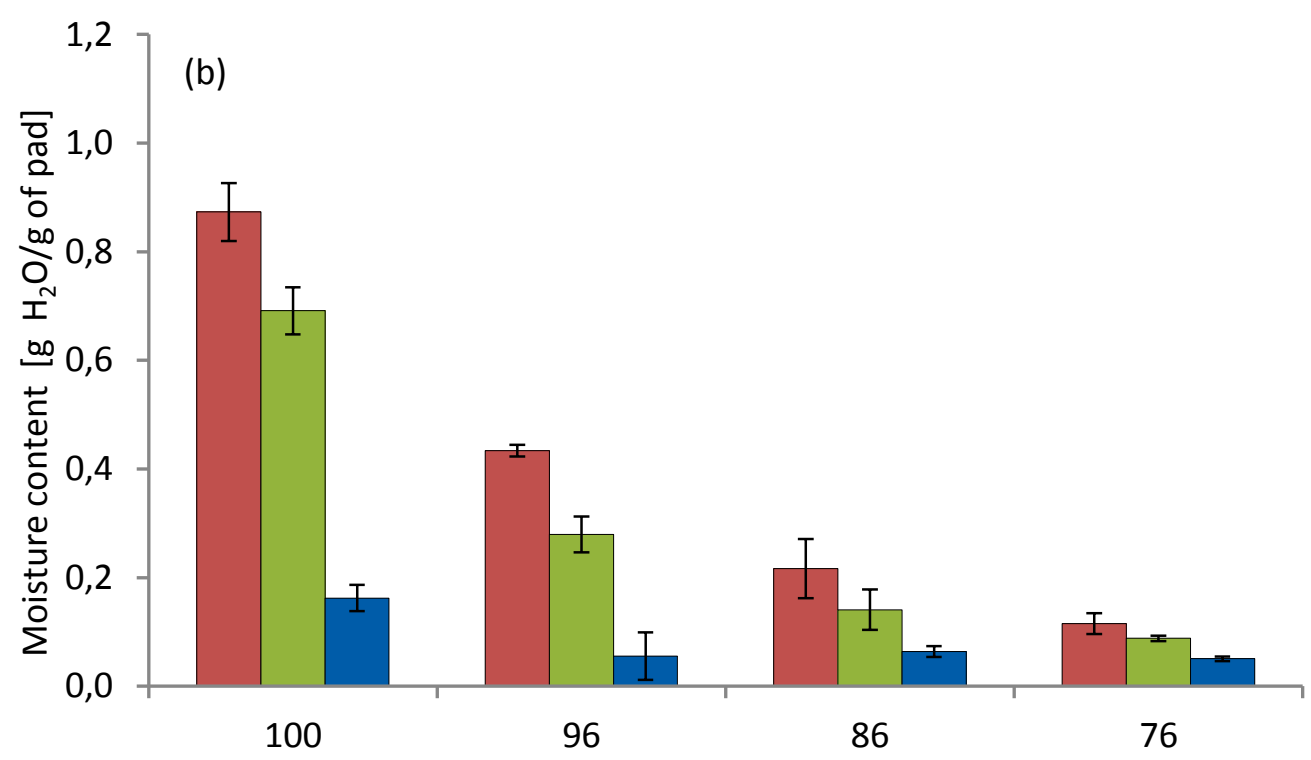

334

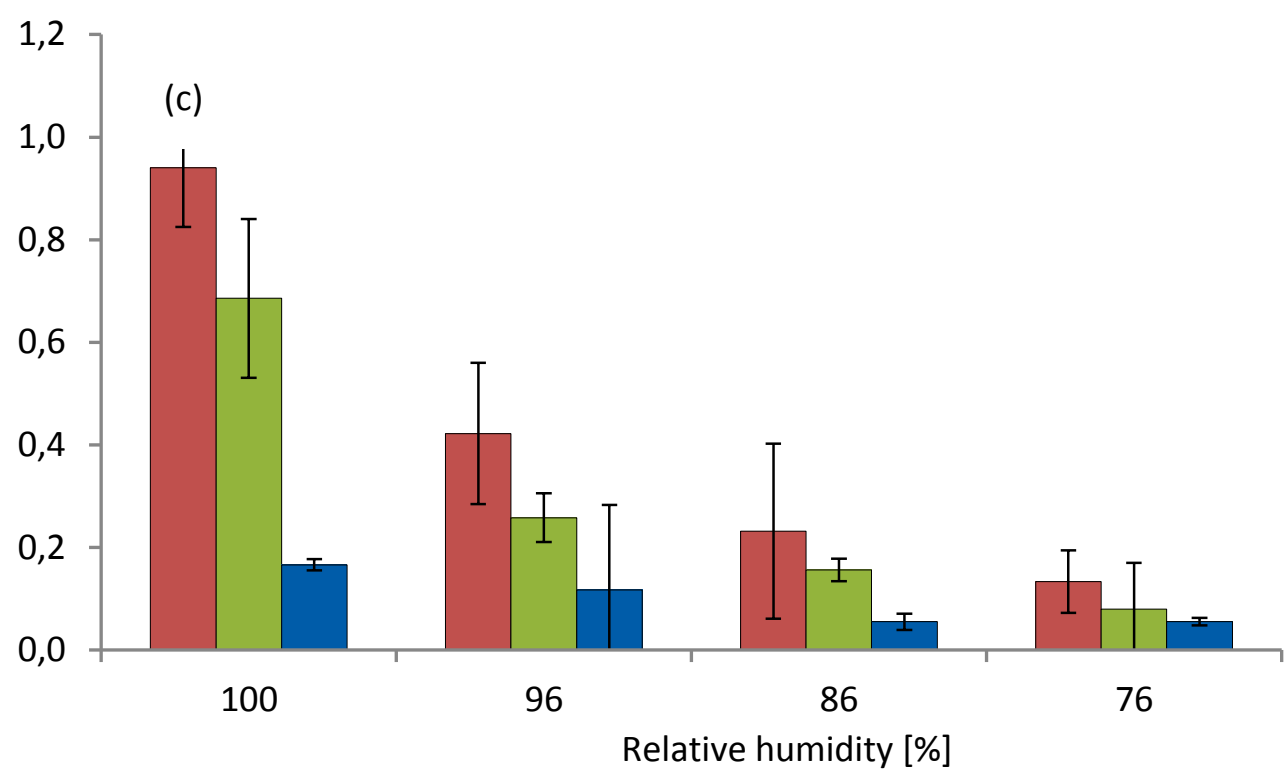

336 Fig 3. Effect of fructose concentration and storage relative humidity on total moisture content $\left(\mathrm{M}_{\mathrm{t}}\right)$ of FruitPad containing different fructose concentration (0: FruitPad00, 20: FruitPad20, and 30\%: 
338 FruitPad30) stored at (a) $4{ }^{\circ} \mathrm{C}$, (b) $12{ }^{\circ} \mathrm{C}$ and (c) $20^{\circ} \mathrm{C}$ for 5 days. Error bars represent standard 339 deviation $(\mathrm{SD})$ of mean values $(\mathrm{n}=3)$.

340

341

342

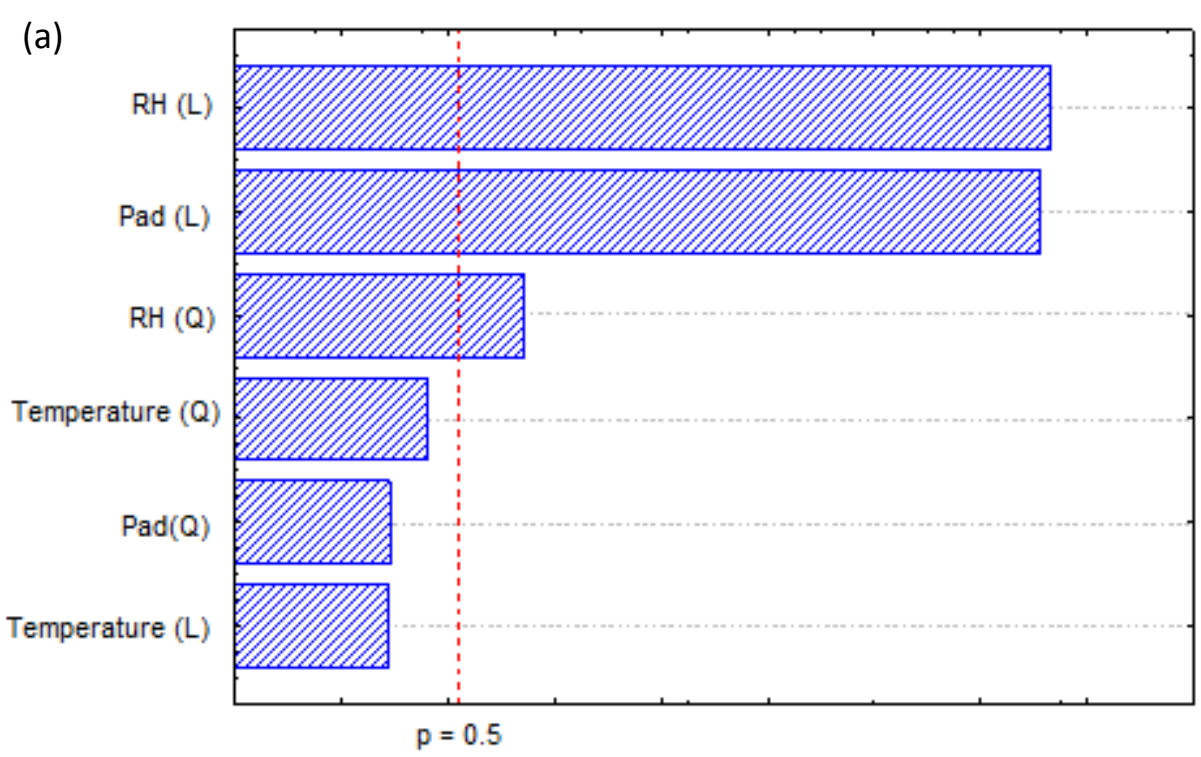

(b)

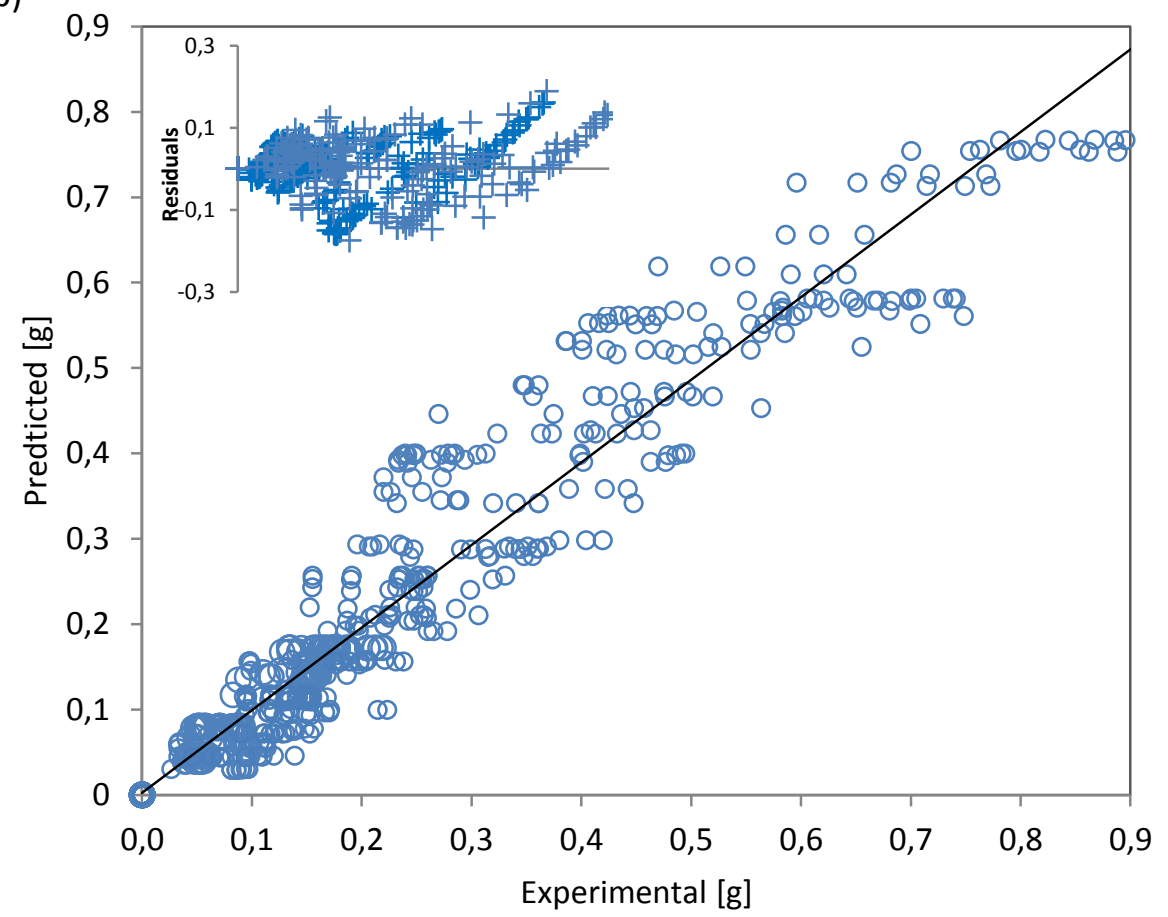

344 Fig 4. Relevant statistical information (a) Pareto analysis of primary model and (b) Experimental vs 345 predicted values of the equilibrium moisture content $(\mathrm{M} \infty)$ of the secondary model for all fructose 346 concentrations (0\%: FruitPad00, 20\%: FruitPad20, and 30\%: FruitPad30). 


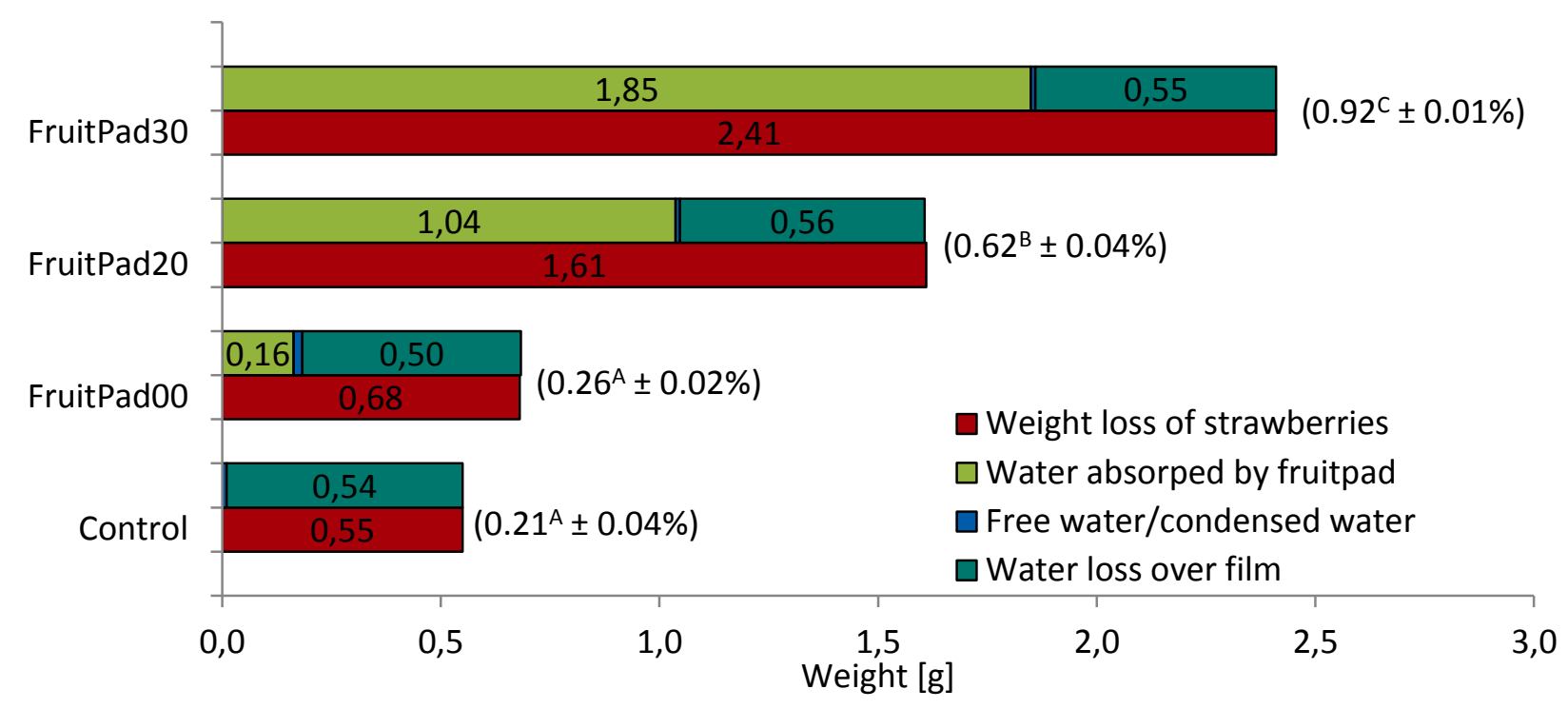

355 Fig 5. In-package moisture dynamics of strawberries packaged with FruitPad containing different 356 fructose concentration (0: FruitPad00, 20: FruitPad20, and 30\%: FruitPad30) stored at $12{ }^{\circ} \mathrm{C}$ for 5 357 days. The values in bracket represent the percentage mean values (mean value \pm standard 358 derivation, $n=2$ ) for total strawberry weight loss. Different upper case superscript is significantly 359 different based on Tukey test at $\mathrm{p}<0.05$. 
Table 1. Estimated parameters of the primary model for FruitPad containing different concentrations of fructose (0\%: FruitPad00, 20\%: FruitPad20, and 30\%: FruitPad30).

\begin{tabular}{|c|c|c|c|c|c|c|c|c|}
\hline \multirow{2}{*}{ Absorbing pad } & \multicolumn{4}{|c|}{$M_{\infty}$} & \multicolumn{4}{|c|}{$\beta_{1}$} \\
\hline & RH: $76 \%$ & $86 \%$ & $96 \%$ & $100 \%$ & $76 \%$ & $86 \%$ & $96 \%$ & $100 \%$ \\
\hline FruitPad00 & 0.0499 & 0.0575 & 0.0886 & 0.1572 & 0.0010 & 0.0100 & 0.3447 & 0.0010 \\
\hline FruitPad20 & 0.0886 & 0.1398 & 0.2656 & 0.5515 & 0.0020 & 0.2741 & 0.5002 & 0.0020 \\
\hline FruitPad30 & 0.1073 & 0.1898 & 0.4118 & 0.6410 & 0.0030 & 0.0100 & 0.8172 & 0.0003 \\
\hline
\end{tabular}

$\mathrm{M}_{\infty}$ is the equilibrium moisture and $\beta_{1}$ is a primary model constant. All parameters shown are at

Table 2. Estimated parameters of the secondary model for FruitPad containing different concentration of fructose (0\%: FruitPad00, 20\%: FruitPad20, and 30\%: FruitPad30).

\begin{tabular}{lllll}
\hline \multirow{2}{*}{ Absorbing pad } & \multicolumn{3}{c}{ Estimated coefficients } & \multirow{2}{*}{$R^{2}(\%)$} \\
\cline { 2 - 4 } & $A$ & $B$ & $\beta_{2}$ & \\
\hline FruitPad00 & 0.00074 & 0.05445 & 0.28333 & 92.56 \\
FruitPad20 & 0.00005 & 0.09371 & 0.77688 & 92.99 \\
FruitPad30 & 0.00031 & 0.07817 & 1.09146 & 96.09 \\
\hline
\end{tabular}

